\title{
Retraction Note to: Correlations of IFN- $\gamma$ genetic polymorphisms with susceptibility to breast cancer: a meta-analysis
}

\author{
Chun-Jiang $\mathrm{Li}^{1,2,4}$ - Yue Dai ${ }^{2}$ Y Yan-Jun $\mathrm{Fu}^{3} \cdot$ Jia-Ming Tian ${ }^{3} \cdot \mathrm{Jin}-\mathrm{Lun} \mathrm{Li}^{4}$. \\ Hong-Jun $\mathrm{Lu}^{3}$ • Feng Duan ${ }^{4}$ Q Qing-Wang $\mathrm{Li}^{1,5}$
}

Published online: 17 August 2015

(C) International Society of Oncology and BioMarkers (ISOBM) 2015

Retraction to: Tumor Biol. (2014) 35:6867-6877

DOI 10.1007/s13277-014-1856-6

The Publisher and Editor retract this article in accordance with the recommendations of the Committee on Publication Ethics (COPE). After a thorough investigation we have strong reason to believe that the peer review process was compromised.

The online version of the original article can be found at http://dx.doi.org/ 10.1007/s13277-014-1856-6.

Qing-Wang Li

liqingwang124@163.com

1 College of Environment and Chemical Engineering, Yanshan University, Hebei Street No. 438, Qinhuangdao 066004, People's Republic of China

2 College of Basic Medicine, Jiamusi University, Jiamusi 154007, People's Republic of China

3 The First Affiliated Hospital of Jiamusi University, Jiamusi 154007 , People's Republic of China

4 The Second Affiliated Hospital of Jiamusi University, Jiamusi 154007, People's Republic of China

5 College of Animal Science, Northwest A\&F University, Xinong Street No. 22, Yangling 712100, People's Republic of China 